\title{
A Measure of the Interference Effect Distribution*
}

\author{
Thibault Gajdos ${ }^{\dagger} \quad$ Mathieu Servant ${ }^{\ddagger} \quad$ Thierry Hasbroucq ${ }^{\S}$ \\ Karen Davrancheף
}

December 19, 2019

\begin{abstract}
We elaborated an index, the Interference Distribution Index, that allows to quantify the relation between response times and the size of the interference effect. This index is associated to an intuitive graphical representation, the Lorenz-interference plot. We show that this index has some convenient properties in terms of sensitivity to changes in the distribution of the interference effect and to aggregation of individual data. Moreover, it turns out that this index is the only one (up to an arbitrary increasing transformation) possessing these properties. The relevance of this index is illustrated through simulations of a cognitive model of interference effects and reanalysis of experimental data.
\end{abstract}

Key words: delta plots, conflict tasks, interference effect, mental chronometry.

\section{Introduction}

Mental chronometry relies on the analysis of response time (RT) across different experimental conditions to make inferences on underlying cognitive processes (Donders, 1868). Beyond comparing mean RT in each experimental condition, one can study RT distributions (Luce, 1986). De Jong, Liang, and Lauber (1994) introduced a particular method, called delta plot, to describe the link between response speed and the size of an experimental effect. This method consists first in computing RT quantiles in each condition, and then plotting their difference against their mean. Using this method, one can see for instance whether the magnitude of an experimental factor is larger for slow than for fast responses.

Delta plots have proven to be specially fruitful in the study of tasks involving a stimulus-response compatibility (SRC) manipulation, such as the Simon task (Simon, 1969). In this task, subjects have to choose between a left- and a right-hand key press according to the color of a visual stimulus presented a few degrees either to the left or

\footnotetext{
*All author contributed equally

${ }^{\dagger}$ Corresponding author. Aix Marseille Univ, CNRS, LPC, Marseille, France. E-mail: thibault.gajdos@univ-amu.fr

${ }^{\ddagger}$ Université de Bourgogne Franche-Comté, Laboratoire de Psychologie, Besançon, France. E-mail: mathieu.servant@univ-fcomte.fr

$\S^{\S}$ Aix Marseille Univ, CNRS, LNC, Marseille, France. E-mail: thierry.hasbroucq@univ-amu.fr

`Aix Marseille Univ, CNRS, LPC, Marseille, France. E-mail: karen.davranche@univ-amu.fr
} 
the right of a fixation point. Mean RTs are typically slower for contralateral stimulusresponse associations (incompatible trials) than for ipsilateral ones (compatible trials). De Jong et al. (1994) found that the spatial correspondence effect (difference between incompatible and compatible trials) was larger for fast responses than for slow ones, resulting in decreasing delta plots.

This result has been consistently replicated (for reviews, see Pratte, Rouder, Morey, \& Feng, 2010; Proctor, Miles, \& Baroni, 2011; Schwarz \& Miller, 2012). Delta plots that level-off for slower RTs, or even become decreasing, are not restricted to the Simon task and have also been observed in other tasks involving some kind of conflict, such as the Eriksen flanker task (e.g. Burle, Spieser, Servant, \& Hasbroucq, 2014; Mattler, 2003; Ridderinkhof, Scheres, Oosterlaan, \& Sergeant, 2005; Wylie, Ridderinkhof, Eckerle, \& Manning, 2007; Wylie et al., 2009), masked priming tasks (Atas \& Cleeremans, 2015; Eimer, 1999; Finkbeiner \& Caramazza, 2008), or tasks involving lying and truth telling (Debey, Ridderinkhof, De Houwer, De Schryver, \& Verschuere, 2015). Such experimental results stand in sharp contrast with the positive linear relation between mean and standard deviation of RTs usually observed (Luce, 1986; Wagenmakers \& Brown, 2007).

These observations suggest that the shape of delta plots might be the signature of some specific cognitive processes. For instance, some argued that it reflects the spontaneous decay of an automatic response (Hommel, 1993, 1994), while others proposed that it results from the deployment of an active inhibitory process (De Jong et al., 1994; Ridderinkhof, 2002a, 2002b). Recently, various cognitive architectures compatible with the particular relation between RT and the size of the interference effect observed in conflict tasks have been proposed (see, e.g., Hübner \& Töbel, 2019; Schwarz \& Miller, 2012; Ulrich, Schröter, Leuthold, \& Birngruber, 2015).

All these theories have in common to propose a cognitive interpretation of distribution of the interference effect. While delta plots provide a compelling visualisation of the relation between RT and the size of the interference effect, it is not obvious how they could be translated into numerical measures suitable for statistical purposes (as required, for instance, to test one of the above mentioned theories). The aim of this article is precisely to solve this issue, by providing a quantitative measure of the distribution of the interference effect. It is important to keep in mind that, by themselves, delta plots say nothing about the cognitive processes at play (Zhang \& Kornblum, 1997). The same is true about the index we propose: it is a measure of the effects of the cognitive processes, not of their nature and causes. After introducing this new index, we will illustrate its properties and compare it to other methods through simulations of a computational model for conflict tasks (Ulrich et al., 2015) and reanalyses of an actual data-set.

\section{The Interference Distribution Index}

\subsection{Definition}

While the following analysis might be applied to data from any type of task, we will consider a task involving an SRC manipulation, such as the Simon task. Formally, let $F$ be the cumulative distribution function of RTs for correct trials for which stimulus and 
response are incompatible, and $G$ be the cumulative distribution function of RTs for correct trials for which stimulus and response are compatible. Given a cumulative distribution function $F$, its associated quantile function is defined as : $F^{-1}(p)=\inf \{x \in \mathbb{R} \mid F(x) \geq p\}$. Let $D(F, G)(p)$ be the size of the interference effect, as measured by the difference of the RTs between conditions at the $p^{\text {th }}$ quantile (i.e., $\left.D(F, G)(p)=F^{-1}(p)-G^{-1}(p)\right)$. The delta plot corresponding to $F$ and $G$ is obtained by plotting $D(F, G)(p)$ against the mean of the RTs in each condition at the $p^{t h}$ quantile (i.e., $M(F, G)(p)=\frac{1}{2}\left[F^{-1}(p)+G^{-1}(p)\right]$ ). When there is no ambiguity about the underlying distribution functions, we will slightly abuse notations, and simply note $M$ and $D$ instead of $D(F, G)$ and $M(F, G)$, respectively. The total interference effect is given by $\mu(D)=\int_{0}^{1} D(p) d p$. We will assume, throughout the remainder of this article, that $\mu(D)>0$.

Our aim is to quantify the relation between RTs and the size of the interference effect. To do so, we will describe how the total interference effect is spread across RT quantiles. Specifically, the proportion of the total interference effect contained in the $p \times 100 \%$ fastest (pairs of) trials is given by:

$$
L(p)=\frac{1}{\mu(D)} \int_{0}^{p} D(x) d x
$$

This function is very similar to the Lorenz curve (Lorenz, 1905). It is noteworthy, however, that a Lorenz curve would require that $D$ be a quantile function, which is not the case here (since it is the difference between two quantile functions). $L(p)$ represents the share of the total interference effect that is contained in the $p \times 100 \%$ fastest (pairs of) trials. Note that $L(0)=0$ and $L(1)=1$. Because of its resemblance with the Lorenz curve, we will call "Lorenz-interference plot" the graphical representation of $L$. If the interference effect is constant across RT trials, then $L(p)=p$ for all $p$, and the Lorenz-interference plot will coincide with the diagonal. The more the interference effect is concentrated among the fastest trials, the higher will be the Lorenz-interference plot. The area under the Lorenzinterference plot is thus a natural measure of the distribution of the interference effect, very much like the Gini index (Gini, 1912) is related to the area under the Lorenz plot. Formally, for any interference effect $D$, we propose the following interference distribution index:

$$
I(D)=\int_{0}^{1} L(p) d p
$$

We call this index the Interference Distribution Index (IDI). Observe that if $D$ is constant, $I(D)=\frac{1}{2}$. On the other hand, if $D$ is decreasing, $I(D)>\frac{1}{2}$. In particular, if all the interference effect is concentrated in the fastest pair of trials, $I(D)=1$. Finally, if $D$ is increasing, $I(D)<\frac{1}{2}$. If all the interference effect is concentrated in the slowest pair of trials, $I(D)=0$. It has sometimes been observed that the interference effect becomes negative at high percentiles (translating into negative delta plots). In such a case, the Lorenz-interference plot will decrease for these highest percentiles. Therefore, these percentiles will contribute negatively to the IDI (because the interference effect will then be less concentrated among slowest trials).

We made available an $\mathrm{R}$ package called RTconflict, that allows to easily implement 
the IDI. ${ }^{1}$ In practice, the quantile functions must be approximated from data. We set the number of quantiles to the number of trials in the condition that contains the smallest number of observations. We then crucially rely on the quantile function in $\mathrm{R}$. This function allows to choose the method used to compute quantiles among the nine methods presented in Hyndman and Fan (1996). We implemented the default method, but allow the user to choose other methods.

\subsection{Properties of the IDI}

We will now turn to some properties of the IDI. First, the IDI only depends on the interference effect, i.e., on the difference between $F^{-1}$ and $G^{-1}$. Thus, it is invariant to any change that does not impact $F^{-1}-G^{-1}$. We call this property "location invariance". Assume, for instance, that for some reason the motor execution time increases in the same way in both conditions for some quantiles. Then the IDI will not be modified.

Next, one can easily see that, if both $F^{-1}$ and $G^{-1}$ are scaled by the same positive constant, the Lorenz-interference plot will not change (because both the numerator and the denominator of $L$ will then be multiplied by the same constant), and so will therefore be the case for the IDI. In other words, the IDI is invariant with respect to a uniform increase of the interference effect across quantiles. We call this property the "scale invariance property". It is an important property, as it allows to measure the distribution of the interference effect, independently from its overall magnitude. This does not imply by any means that the overall interference effect level is not meaningful, but that it should be disentangled from its distributional properties.

The IDI has another invariance property, that allows an easy measure of the distribution of aggregated individual interference effects. Let us consider two interference effects, $D_{1}$ and $D_{2}$, with the same total magnitude (i.e., $\left.\mu\left(D_{1}\right)=\mu\left(D_{2}\right)\right)$. Then, it is easy to check that the IDI corresponding to the weighted average of $D_{1}$ and $D_{2}$ is simply the weighted average of each IDI. Now, what if $\mu\left(D_{1}\right) \neq \mu\left(D_{2}\right)$ ? Recall that we are interested in the distribution of the interference effect, and not in its level. We can thus normalise $D_{1}$ and $D_{2}$ by $\mu\left(D_{1}\right)$ and $\mu\left(D_{2}\right)$, respectively, and aggregate them linearly. We then obtain:

$$
D=\frac{1}{2} \frac{D_{1}}{\mu\left(D_{1}\right)}+\frac{1}{2} \frac{D_{2}}{\mu\left(D_{2}\right)} .
$$

By the above property, we get:

$$
I(D)=\frac{1}{2} I\left(\frac{D_{1}}{\mu\left(D_{1}\right)}\right)+\frac{1}{2} I\left(\frac{D_{2}}{\mu\left(D_{2}\right)}\right) .
$$

Finally, by the scale invariance property, we obtain:

$$
I(D)=\frac{1}{2} I\left(D_{1}\right)+\frac{1}{2} I\left(D_{2}\right) .
$$

In other words, the aggregated IDI is nothing but the average of individual IDIs. We call this property the "aggregation property".

\footnotetext{
${ }^{1}$ This package is available at the following address: https://github.com/thibault-gajdos/RTconflict, and can be installed through devtools: :install_github("thibault-gajdos/RTconflict").
} 
Yet, all this tells little about how the IDI reflects the relation between RT and the size of the interference effect. In that respect, the IDI has a fundamental property, that we call the "transfer property". Assume that the level of interference effect increases for some quantile $p$, and decreases of the exact same amount for some higher quantile $q$. Such a transformation leaves the overall interference effect unchanged, but more concentrated among fastest trials. It is easy to see that such a transformation indeed results in an increase of the IDI. Moreover, the larger the distance between $p$ and $q$, the larger is this increase. On the other hand, the variation of the IDI is the same wherever this change occurs in the interference effect distribution (given the distance between $p$ and $q$ ).

Finally, the IDI is a continuous function. This means that small changes in the RT distributions will result in small changes in the IDI. This property, while a bit technical, has its importance: it means that the IDI will not overreact to small measurement errors.

Importantly, it turns out that not only the IDI has all the properties described above, but it is the only index (up to an increasing transformation) that satisfy them all. In other words, if another index were to satisfy all these properties, it would be an increasing transformation of the IDI. This fact is proven in Appendix A.

\subsection{Alternative Measures of the Interference Effect Distribution}

To the best of our knowledge, mainly two methods are currently used to measure the distribution of the interference effect, and both are directly based on delta plots. A very common method consists in quantifying the decay of the interference effect by the opposite of the slope of the last segment of the delta plot (Delta Slope Index, DSI) (Forstmann, van den Wildenberg, \& Ridderinkhof, 2008; Ridderinkhof, 2002a; van den Wildenberg et al., 2010). This method has been used to determine the effects of various psychiatric or neurodegenerative disorders on inhibitory control mechanisms, such as attention deficit/hyperactivity disorder (e.g. Ridderinkhof et al., 2005; Suarez et al., 2015), Tourette's syndrome (e.g. Wylie, Claassen, Kanoff, Ridderinkhof, \& van den Wildenberg, 2013), alien hand syndrome (McBride, Sumner, Jackson, Bajaj, \& Husain, 2013), Parkinson's disease (e.g. van Wouwe et al., 2016; Wylie et al., 2010), mild cognitive impairment (Wylie et al., 2007) and their modulation by psychotropic drugs (e.g. van Wouwe et al., 2016; Wylie et al., 2012). It has also been used to study the development of inhibitory control across the lifespan (e.g. Ambrosi, Servant, Blaye, \& Burle, 2019; Bub, Masson, \& Lalonde, 2006; de Bruin \& Della Sala, 2017; Joyce, Smyth, Donnelly, \& Davranche, 2014), the effect of hypoxia (Davranche et al., 2016), acute exercise (e.g. Davranche, Hall, \& McMorris, 2009), working memory capacity (Meier \& Kane, 2015), and social context (Belletier et al., 2015; Sharma, Booth, Brown, \& Huguet, 2010).

The DSI satisfies scale invariance, but not location invariance. In particular, a similar (not necessarily uniform) increase of both $F^{-1}$ and $G^{-1}$ (which lets the interference effect unchanged) would actually yield a decrease of the DSI. This index also does not satisfy the aggregation property, which means that the average of the DSI across subjects is difficult to interpret. These difficulties can be circumvented by considering the quantile version of DSI, i.e., by considering the slope of the last segment of a discretised version of $D$. However, neither the DSI, nor its quantile version satisfy the principle of transfer, 
as they are totally insensitive to any change in the early quantiles of the RT distribution. Finally, it should be stressed that the DSI requires a discretisation of the RTs, which entails a substantial loss of information. Moreover, the number of bins considered is quite arbitrary, and might affect the results, as illustrated in Section 3.2.

Another, albeit less frequent method, consists in measuring the distribution of the interference effect by the opposite of the trend of the delta plot (Delta Trend Index, DTI), as estimated by a linear regression (De Jong et al., 1994; Pratte et al., 2010). As the DSI, and for the same reasons, the DTI satisfies the scale invariance property, but neither location invariance nor the aggregation property. It does, however, satisfy the transfer property. One might thus prefer to replace it by the trend of $D$. Formally, let $T(D)$ be the opposite of the trend of $D$, as estimated by an OLS regression. It is obvious that $T$ does not satisfy the invariance property. This can however easily be fixed by normalising it by $\mu(D)$. We then obtain the following Interference Trend Index (ITI):

$$
I T I=\frac{T(D)}{\mu(D)} .
$$

This index satisfies all the properties of the IDI, and is actually equivalent to it: as shown in Appendix B, it is an affine transformation of the IDI. Note that the ITI is slightly distinct from the usual measure of the trend of the delta plot in three respects: first, it is the trend of the interference effect; second, it is computed on all quantiles (instead of a subset of them, e.g., deciles, thereby avoiding the issue of discretisation); third, it is normalised by the mean interference effect. Among other things, this means that the average of individual ITIs can easily be interpreted, contrary to that of delta plot trends.

\section{Illustrations}

In order to better understand the differences between the IDI and the DSI, we will compare the results of these methods on different sets of data. First, we will turn to data generated by a computational model for conflict tasks. This will allow us to see how various cognitive processes, as defined within this model, impact these measures. Next, we will reanalyse experimental data from a study on the impact of cognitive fatigue on cognitive control (Joyce et al., 2014). ${ }^{2}$

\subsection{Diffusion Model for Conflict Tasks}

The Drift Diffusion Model (DDM, Ratcliff, 1978) is one of the most popular computational model of RTs in two-alternative forced choice. It essentially postulates that the evidence favouring an option is accumulated until it reaches a threshold, at which stage a decision is made. While the DDM proved to be very successful in modelling RTs in many paradigms, it has been shown that it cannot account for decreasing delta slopes (Pratte et al., 2010; Schwarz \& Miller, 2012).

The Diffusion Model for Conflict Tasks (DMC, Ulrich et al., 2015) is a versatile diffusion model that can account for delta plots that level-off, or even get decreasing, as observed

\footnotetext{
${ }^{2}$ All codes and data needed to reproduce the results of this section are available at https://osf.io/5r8mq/.
} 
in experiments involving SRC manipulations, such as the Simon task. The DMC is an extension of the DDM, where the amount of evidence in favour of one option results from the sum of two diffusion processes: a controlled process, with a constant drift rate $\left(\mu_{c}\right)$, and an automatic process, which generates an expected accumulated evidence that increases in favour of one option (the response compatible to the stimulus) before progressively fading. This dynamics is described by a rescaled Gamma density function, with a peak amplitude $A$, a shape parameter $\alpha$ and a characteristic time $\tau .^{3}$ The peak amplitude determines the strength of the automatic activation, while the shape parameter and characteristic time are related to the speed of the fading of automatic activation, be it due to an active suppression or a passive decay. The shape parameter is generally fixed at 2 (Ulrich et al., 2015). With this parameterisation, the characteristic time corresponds to the peak latency of the automatic activation. More precisely, the smaller the parameter $\tau$, the faster the decline of the automatic activation.

We simulated the DMC for various values of $\tau$, and computed the IDI and DSI for each simulated data set. The DSI was calculated from deciles of the delta plots. Observe that the IDI and the DSI are supposed to vary in the same direction, i.e., to be larger whenever the interference effect is more concentrated among the fastest responses. We thus expected these indices to mainly be impacted by the characteristic time, and, specifically, to decrease when $\tau$ increases.

We kept the parameters related to the controlled processes and the speed-accuracy strategy fixed, with values chosen in a plausible range (Servant, White, Montagnini, \& Burle, 2016; Ulrich et al., 2015; White, Servant, \& Logan, 2017), while letting the peak amplitude and the characteristic time varying (see legend of Figure 1 for more details).

\footnotetext{
${ }^{3}$ The expected mean of the accumulated evidence generated by the automatic process at time $t$ is thus given by: $A e^{-\frac{t}{\tau}}\left[\frac{t e}{(\alpha-1) \tau}\right]^{\alpha-1}$.
} 

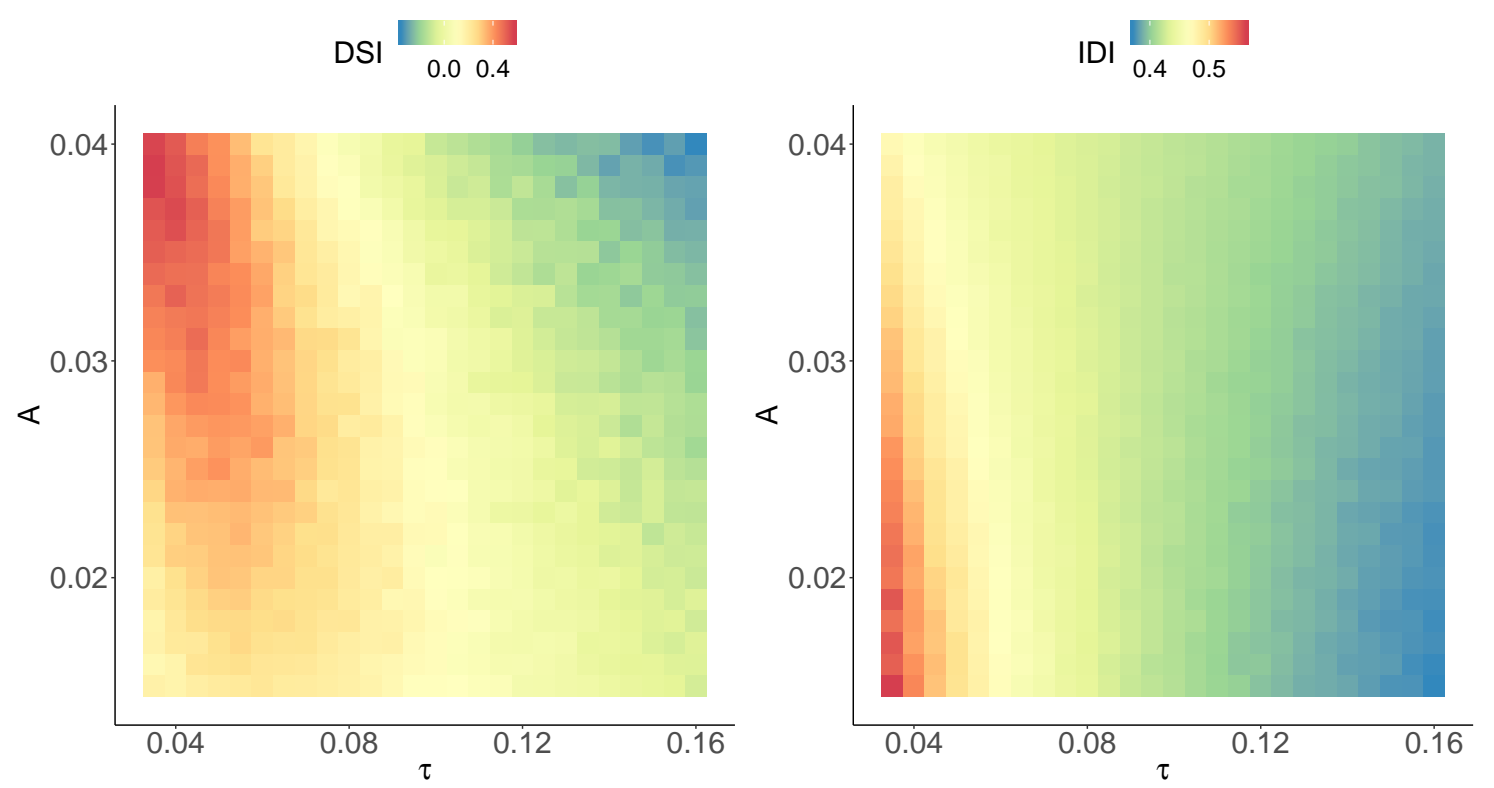

Figure 1: Heat maps representing the values of the Delta Slope Index (left) and the Interference Distribution Index (right) for simulated data from DMC as a function of the peak amplitude $A$ and the characteristic time $\tau$. A varies between 0.015 and 0.04 (by steps of 0.001 ), and $\tau$ varies between 0.035 and 0.160 (by steps of 0.005 ). Others parameters are kept fixed with the following values: $\mu_{c}=0.5, b=0.05, \mathrm{Ter}=.03, \alpha=2$. The model is simulated in seconds, with a diffusion coefficient equal to 0.1 . For each set of parameter values, 160000 trials were simulated.

Figure 1 clearly shows that the DSI is not a very good measure of the interference effect distribution. It is strongly affected by the peak of the automatic activation $A$, and is not monotone with respect to the characteristic time. This implies that there is a range of values of $\tau$ such that, for a fixed $A$, the DSI increases with $\tau$, which is exactly the opposite to what is expected. The IDI seems, in that respect, more satisfactory, as it globally decreases when $\tau$ increases, and is much less sensitive to variations of $A$.

In order to further illustrate the differences between the IDI and the DSI, we plot the cumulative distributions for compatible and incompatible trials, delta plots, and Lorenzinterference plots for two sets of parameters (see the legend of Figure 2 for details). As $\tau$ increases, the interference effect is more concentrated among slowest trials. This is clearly apparent when inspecting the cumulative distributions for compatible and incompatible trials in Figure 2: the distance between the cumulative distributions increases more in the bottom panel (corresponding to $\tau=0.06$ ) than in the top panel (corresponding to $\tau=0.03)$. The Lorenz-interference plots confirm this fact. For instance, the $50 \%$ fastest trials represent $52 \%$ of the interference effect when $\tau=0.03$, while they represent only $40 \%$ of the interference effect when $\tau=0.06$. As a consequence, the IDI is larger for $\tau=0.03$ (IDI $=0.52$ ) than for $\tau=0.06$ (IDI $=0.44)$. Yet, the DSI index is smaller for $\tau=0.03(\mathrm{DSI}=0.29)$ than for $\tau=0.06(\mathrm{DSI}=0.37)$. The reason for this result appears clearly when one inspects the delta plots. While the slope of the last segment is indeed steeper for $\tau=0.06$, it becomes decreasing later (for the ninth decile, instead of the sixth decile for $\tau=0.03)$. The DSI fails to take into account this difference. 

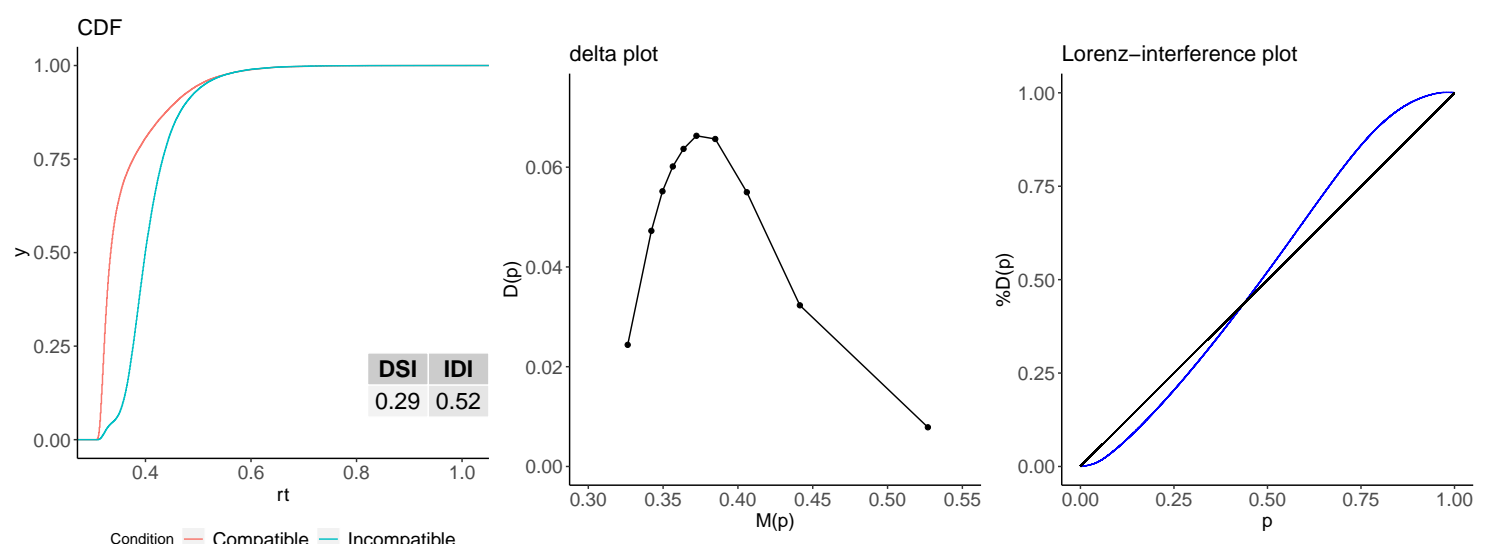

$$
\tau=0.06
$$
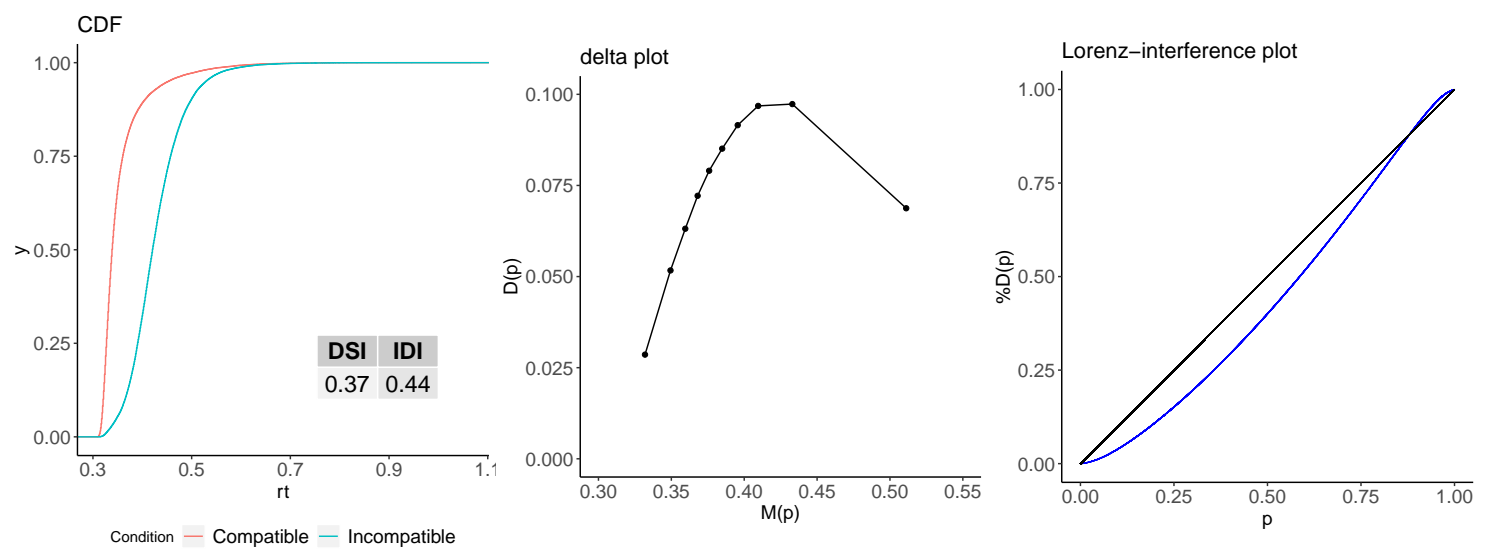

Figure 2: Cumulative distributions for compatible and incompatible trials (left), delta plots (middle), and Lorenz-interference plots (right) for two characteristic times: $\tau=0.03$ (top) and $\tau=0.06$ (bottom), and $A=0.03 .100000$ trials were simulated for each value of $\tau$. Other parameters were kept identical to those in Figure 1. DSI and IDI are displayed with the cumulative distribution functions.

\subsection{Cognitive Fatigue and Control}

We now turn to an actual data set, in order to investigate to what extent the choice of an index of the interference effect distribution might lead to different conclusions. Joyce et al. (2014) investigated the impact of cognitive fatigue (measured by time on task) on control, defined as the suppression of an automatic activation. We reanalyse here their results. During two experimental sessions, twenty-four subjects completed four sets of five blocks of 96 trials (i.e., 1920 trials of the Simon task per session). We group trials of both sessions in two conditions: the first two sets (condition "early") and the last two sets 
(condition "late"). We expect to find that fatigue induces a depletion of cognitive control. According to Ridderinkhof (2002a) activation-suppression theory, the interference effect should be more concentrated among fast trials in the "early" than in the "late" condition. We compare the DSI and the IDI across conditions using a Bayesian parameter estimation (Kruschke, 2013), implemented with the R package BEST (Kruschke \& Meredith, 2018). We use BEST default priors with 10000 burn-in steps, and report 95\% highest-density intervals (HDI).

We start with the DSI. The comparison of slopes requires to decide the number of quantiles used to compute the slopes. We first compare slopes between the last two deciles (see Figure 3, left panel), and observe that there is no significant difference between the two conditions (early condition: DSI $=0.11$, late condition: $\mathrm{DSI}=0.061$, HDI of the difference: $[-0.03,0.12])$. On the other hand, comparing the slopes between the last quintiles reveals a better control for the early condition (early condition: DSI $=0.13$, late condition: DSI $=0.051$, HDI of the difference: $[0.020,0.13])$. These results show that the DSI might lead to different conclusion depending upon the number of quantiles considered (which is an arbitrary choice).

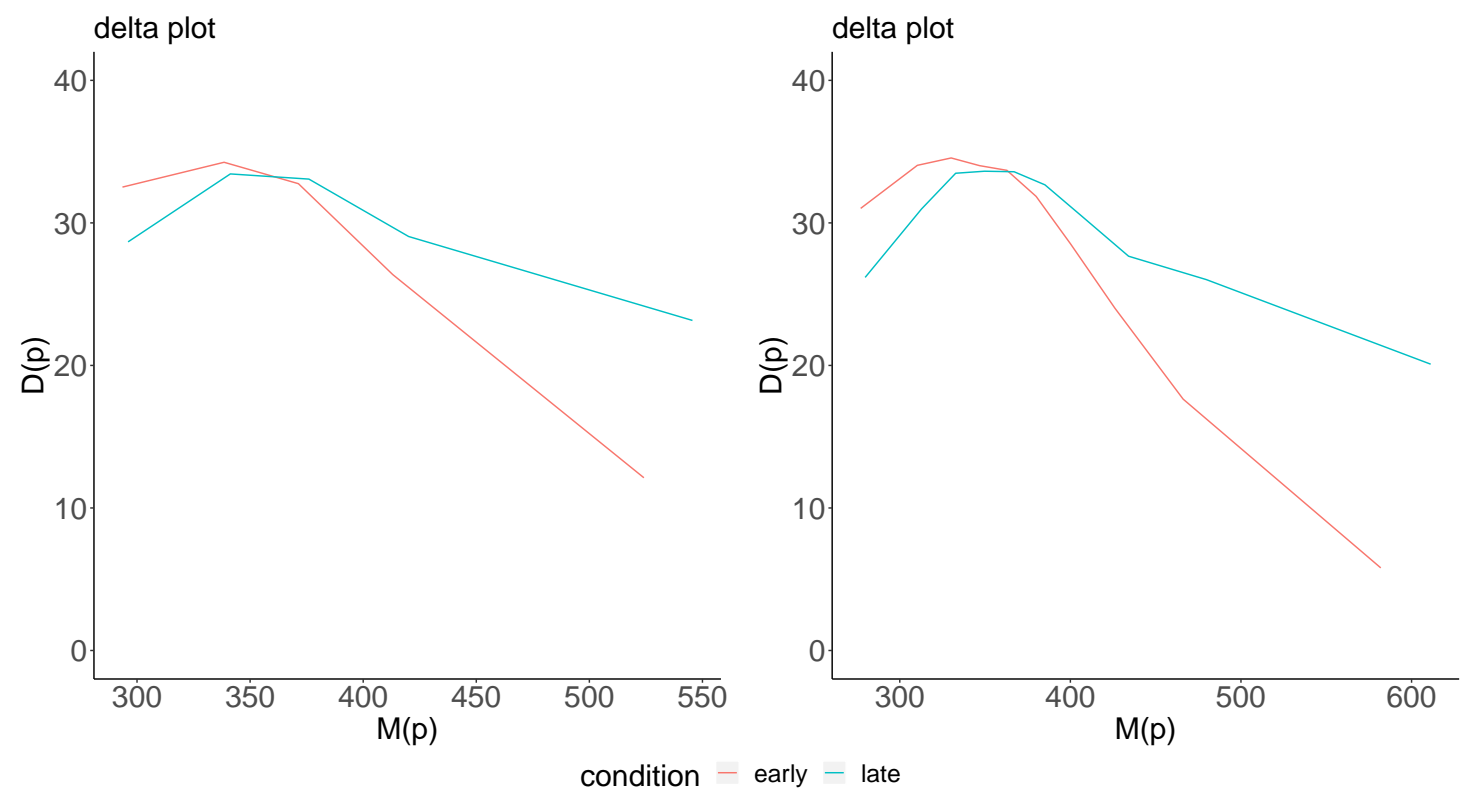

Figure 3: Delta plots in the early condition (red) and late conditions (blue) averaged across subjects, for 5 (left panel) and 10 (right panel) bins.

We now turn to the IDI. Interference effects $(D)$ and Lorenz-interference plots are shown in Figure 4, where group interference plots are obtained by computing the average of individual ones. The IDI index reveals a better control in the early condition compared to the late condition (early condition: $I D I=0.61$, late condition: $I D I=0.51$, HDI of difference : $[0.001,0.096])$, suggesting that time-on-task might impair cognitive control, as expected. 

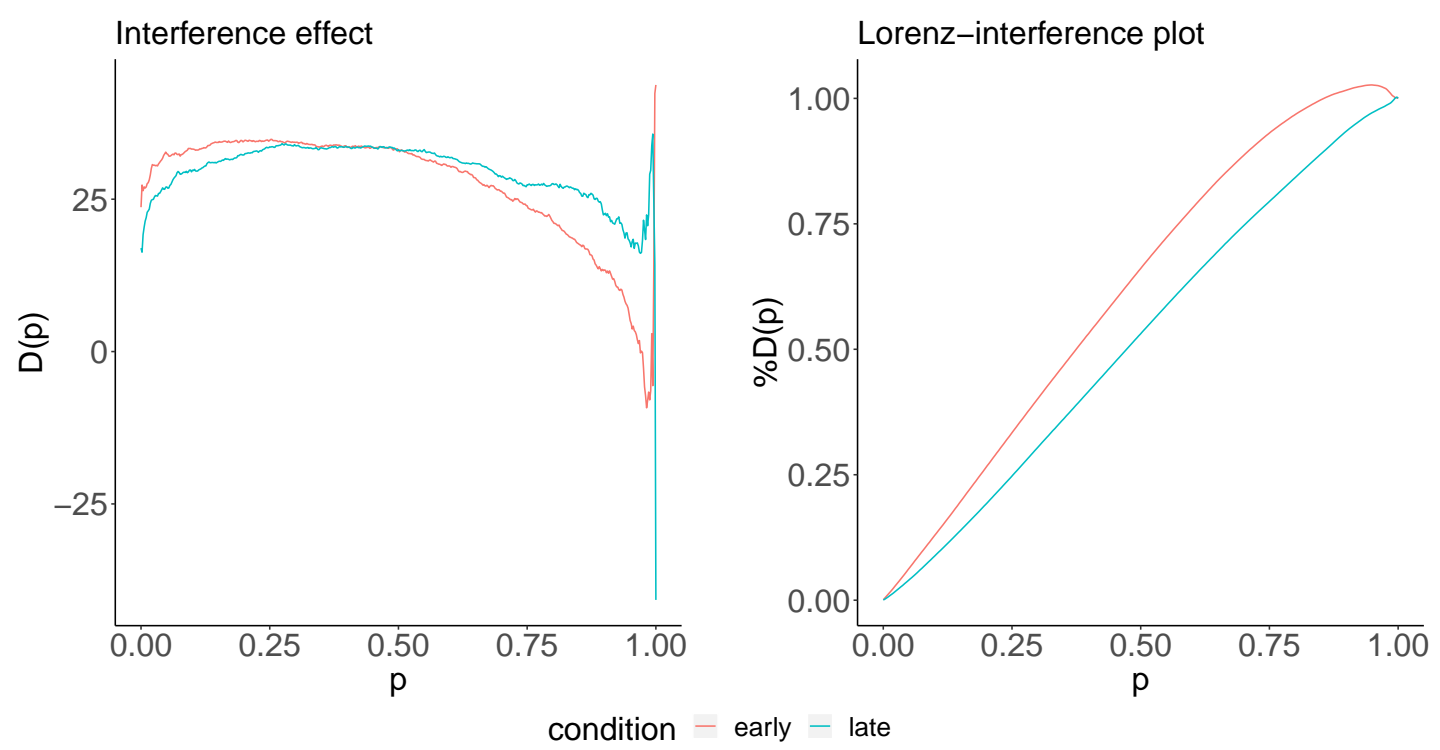

Figure 4: Interference effect (representing the interference effect at each quantile, left) and Lorenz-interference plots (representing the share of the mean interference effect that is contained in the $p \times 100 \%$ fastest trials, right) in the early (red) and late (green) conditions. The Lorenz-interference plot corresponding to early condition (red) is below the Lorenz-interference plot corresponding to late condition (green), which corresponds to a larger IDI in the early condition as compared to the late condition.

\section{Discussion}

Delta plots are a natural way to represent the link between response speed and the size of an experimental effect, by plotting the difference between RT quantiles in each experimental factor against their means. It has been shown that, while they are generally linearly increasing, they can sometimes deviate from this pattern in tasks involving SRC effects, such as the Simon task (De Jong et al., 1994). This observation lead to interpret the distribution of the interference effect in SRC tasks as the signature of some specific cognitive processes (De Jong et al., 1994; Pratte et al., 2010; Proctor et al., 2011; Ridderinkhof, 2002a, 2002b; Schwarz \& Miller, 2012; Ulrich et al., 2015). In that context, it is important to be able to measure the distribution of the interference effect, independently of any cognitive theory.

In this article, we aimed at providing such a measure. While extremely useful as a graphical device, delta plots are not a pure representation of the distribution of the interference effect, as they also account for the average response time at each quantile. We thus step back from the delta plots to the distribution of the interference effect, and wonder how it can be quantified. We proposed a simple measure (the Interference Distribution Index, IDI), that is essentially a concentration index, measuring to what extent the interference effect is concentrated among the fastest trials. It is associated to a graphical representation (the Lorenz-interference effect), that represents the proportion of the total interference effect concentrated within the $p \%$ fastest trials. We show that this measure has several convenient properties, in terms of invariance with respect to scale transformation, sensitivity to increase of concentration among fastest trials, and aggregation of individual data. Moreover, we show that this measure is the only one 
(up to an arbitrary increasing transformation) that possesses all these properties. We also show how this measure can be applied to specific computational models and actual experimental data.

It should be noted that we have restricted attention, throughout this article, to the interference effect among correct trials. The rationale for this choice is that the amount and timing of errors are likely to reflect different cognitive mechanisms than the distribution of the interference effect. In particular, it has been argued that the timing of errors could be the signature of the strength of response capture (see van den Wildenberg et al., 2010, for a review), and an index dedicated to measure this effect has been proposed (Servant, Gajdos, \& Davranche, 2018). It must also be stressed that, by construction, the IDI does not depend of the size of the interference effect. Again, this does not mean that this information is not important, but simply that we tried to design an index that specifically measures the distribution of the interference effect. The specificity of chronometric indices might help disentangle different important aspects of cognitive processes.

Finally, it might be worth noting that, while new, the IDI is closely related to the intuition of the authors who first proposed to use delta plots to quantify the distribution of the interference effect (De Jong et al., 1994). Indeed, these authors suggested to use the trend of the delta plot, as approximated by a linear regression, as a measure of the distribution of the interference effect. It turns out that this measure does not allow a simple aggregation across individuals (because it relies on delta plots), and does not satisfy a simple invariance property with respect to changes that do not impact the interference effect (location invariance). However, these issues can easily be solved by considering the trend of the interference effect instead of that of the delta plot. Yet, this measure would depend on the overall interference effect. To solve this problem, one can simply normalise it by the total interference effect. It turns out that the resulting measure (the ITI) is equivalent to the IDI. In other words, the present work can be seen as a rationalisation of the use of the trend of the interference effect as a measure of its distribution. As a side result, it comes with an intuitive graphical representation (the Lorenz-interference plot).

\section{A Axiomatic characterization of the Interference Distribu- tion Index}

We provide an axiomatic characterization for the Interference Distribution Index. In order to do so, we will formally state the properties exposed in Subsection 2.2, and show that the IDI is the only index that satisfy them all.

Let us first introduce some notations and definitions. Let $\mathscr{F}$ be the set of strictly increasing continuous cumulative distribution functions of bounded response times. Note that for any $F$ in $\mathscr{F}$, its inverse $F^{-1}$ is continuous, non-negative and bounded. The set of interference effects is thus $\mathscr{D}=\left\{F^{-1}-G^{-1} \mid F, G \in \mathscr{F}\right.$ and $\left.\int_{0}^{1} F^{-1}(p)-G^{-1}(p) d p>0\right\}$. We recall that, for any $D \in \mathscr{D}, \mu(D)=\int_{0}^{1} D(p) d p$. One easily checks that for all $\lambda>0$ and all $D \in \mathscr{D}, \lambda D \in D$, and for all $\alpha \in[0,1], D_{1}, D_{2} \in D, \alpha D_{1}+(1-\alpha) D_{2} \in \mathscr{D}$.

We now introduce the properties presented in sub-section 2.2 formally as axioms. In what follows, $I$ is an IDI index. 
Axiom 1. The IDI I is a continuous real function on $\mathscr{D}$.

Axiom 2. For all $\lambda>0$ and $D \in \mathscr{D}, I(\lambda D)=I(D)$.

Axiom 3. For all $D_{1}, D_{2} \in \mathscr{D}$ such that $\mu\left(D_{1}\right)=\mu\left(D_{2}\right)$, all $\alpha \in[0,1]$,

$$
I\left(\alpha D_{1}+(1-\alpha) D_{2}\right)=\alpha I\left(D_{1}\right)+(1-\alpha) I\left(D_{2}\right) .
$$

Axiom 4. For all $D_{1}, D_{2}, 0 \leq q<r \leq 1$ and $\varepsilon>0$ such that:

$$
\begin{aligned}
& \left\{\begin{array}{l}
D_{1}(q)=D_{2}(q)+\varepsilon \\
D_{1}(r)=D_{2}(r)-\varepsilon \\
D_{1}(p)=D_{2}(p) \text { for all } p \notin\{q, r\},
\end{array}\right. \\
& I\left(D_{1}\right)>I\left(D_{2}\right) .
\end{aligned}
$$

Axiom 5. For all $D_{1}, D_{2}, 0 \leq q_{1}<r_{1} \leq 1,0 \leq q_{2}<r_{2} \leq 1$ satisfying $r_{1}-q_{1}=r_{2}-q_{2}$, and $\varepsilon>0$ such that:

$$
\left\{\begin{array}{l}
D_{1}\left(q_{1}\right)=D_{2}\left(q_{1}\right)+\varepsilon \\
D_{1}\left(r_{1}\right)=D_{2}\left(r_{1}\right)-\varepsilon \\
D_{1}\left(q_{2}\right)=D_{2}\left(q_{2}\right)-\varepsilon \\
D_{1}\left(r_{2}\right)=D_{2}\left(r_{2}\right)+\varepsilon \\
D_{1}(p)=D_{2}(p) \text { for all } p \notin\left\{q_{1}, r_{1}, q_{2}, r_{2}\right\}, \\
\qquad I\left(D_{1}\right)=I\left(D_{2}\right) .
\end{array}\right.
$$

Theorem 1. An IDI I satisfies Axioms 1 to 5 if, and only if,

$$
I(D)=\int_{0}^{1} L(D)(p) d p
$$

up to a strictly increasing transformation.

Proof. Necessity part of the theorem is easily checked. We thus only provide a (sketch of the) proof for its sufficiency part.

Let us define $J(D)=\mu(D) I(D)$. We will show that $J$ is affine, i.e., $J\left(\alpha D_{1}+(1-\right.$ $\left.\alpha) D_{2}\right)=\alpha J\left(D_{1}\right)+(1-\alpha) J\left(D_{2}\right)$ for all $\alpha \in[0,1]$. Let $D_{1}, D_{2} \in \mathscr{D}$ and $\alpha \in[0,1]$. Define $m=\alpha \mu\left(D_{1}\right)+(1-\alpha) \mu\left(D_{2}\right)$. We have:

$$
\begin{aligned}
J\left(\alpha D_{1}+(1-\alpha) D_{2}\right) & =m I\left(\alpha D_{1}+(1-\alpha) D_{2}\right), \text { by definition of } J \\
& =m I\left(\frac{\alpha}{m} D_{1}+\frac{1-\alpha}{m} D_{2}\right), \text { by Axiom } 2 \\
& =m I\left(\frac{\alpha \mu\left(D_{1}\right)}{m} \frac{D_{1}}{\mu\left(D_{1}\right)}+\frac{(1-\alpha) \mu\left(D_{2}\right)}{m} \frac{D_{2}}{\mu\left(D_{2}\right)}\right) \\
& =m\left[\frac{\alpha \mu\left(D_{1}\right)}{m} I\left(\frac{D_{1}}{\mu\left(D_{1}\right)}\right)+\frac{(1-\alpha) \mu\left(D_{2}\right)}{m} I\left(\frac{D_{2}}{\mu\left(D_{2}\right)}\right)\right], \text { by Axiom } 3 \\
& =m\left[\frac{\alpha \mu\left(D_{1}\right)}{m} I\left(D_{1}\right)+\frac{(1-\alpha) \mu\left(D_{2}\right)}{m} I\left(D_{2}\right)\right], \text { by Axiom } 2
\end{aligned}
$$




$$
\begin{aligned}
& =\alpha \mu\left(D_{1}\right) I\left(D_{1}\right)+(1-\alpha) \mu\left(D_{2}\right) I\left(D_{2}\right) \\
& =\alpha J\left(D_{1}\right)+(1-\alpha) J\left(D_{2}\right), \text { by definition of } J .
\end{aligned}
$$

Therefore, $J$ affine. It is also continuous because $I$ is continuous. Note that $J(0)=0$, and that by Axiom 4, $J(D)>0$ for all $D>0$. We consider the linear extension $\tilde{J}$ of $J$ on the vector space spanned by $\mathscr{D}$. It is easily checked that $\tilde{J}$ is positive continuous linear. Thus, by the Riesz Representation Theorem (Aliprantis \& Border, 2006, chap. 13), there exists a unique continuous and bounded function $\varphi$ on $[0,1]$ such that for all $D \in \mathscr{D}$,

$$
\tilde{J}(D)=\int_{0}^{1} \varphi(p) D(p) d p
$$

We now consider $J$ as the restriction of $\tilde{J}$ on $\mathscr{D}$. Axioms 4 and 5 imply that $\varphi$ is a decreasing affine function (Gajdos, 2002, theorem 3.1). Therefore, there exist $a<0$ and $b \in \mathbb{R}$ such that:

$$
J(D)=\int_{0}^{1}(a p+b) D(p) d p .
$$

Integrating (1) by part we obtain:

$$
J(D)=(a+b) \mu(D)-a \int_{0}^{1} \int_{0}^{p} D(t) d t d p .
$$

Thus, dividing both terms of the above equation by $\mu(D)$ we get:

$$
I(D)=(a+b)-a \int_{0}^{1} L(p) d p .
$$

Finally, the coefficients $a$ and $b$ can be arbitrarily chosen. Setting $a=-1$ and $b=1$ yields to:

$$
I(D)=\int_{0}^{1} L(D)(p) d p
$$

\section{B Equivalence between IDI and ITI}

Let $P$ be defined on $[0,1]$ by $P(p)=p$, and consider $P$ and $D$ as random variables on $[0,1]$ with respect to the uniform distribution. Then, for all $D \in \mathscr{D}$,

$$
T(D)=-\frac{\operatorname{cov}(D, P)}{\operatorname{var}(P)} .
$$

We have:

$$
\begin{aligned}
\operatorname{cov}(D, P) & =\int_{0}^{1} p D(p) d p-\int_{0}^{1} p d p \int_{0}^{1} D(p) d p \\
& =\frac{1}{2} \mu(D)-\mu(D) \int_{0}^{1} D(p) d p, \text { by integration by parts } \\
& =\frac{1}{2} \mu(D)-\mu(D) I(D), \text { by definition on } I .
\end{aligned}
$$


Since $\operatorname{var}(P)=\frac{1}{12}$, we obtain:

$$
\operatorname{ITI}(D)=12 I(D)-6
$$

Therefore, ITI and IDI are equivalent (up to an affine transformation).

Open Practices Statement: All the material used in this article is available at https://osf.io/5r8mq/ (script and data) and https://github.com/thibault-gajdos/RTconflict (R package).

\section{References}

Aliprantis, C. D., \& Border, K. C. (2006). Infinite dimensional analysis: A hitchhiker's guide. Springer, Berlin.

Ambrosi, S., Servant, M., Blaye, A., \& Burle, B. (2019). Conflict processing in kindergarten children: New evidence from distribution analyses reveals the dynamics of incorrect response activation and suppression. Journal of Experimental Child Psychology, 177, $36-52$.

Atas, A., \& Cleeremans, A. (2015). The temporal dynamic of automatic inhibition of irrelevant actions. Journal of Experimental Psychology: Human Perception and Performance, 41(2), 289.

Belletier, C., Davranche, K., Tellier, I. S., Dumas, F., Vidal, F., Hasbroucq, T., \& Huguet, P. (2015). Choking under monitoring pressure: Being watched by the experimenter reduces executive attention. Psychonomic bulletin \& review, 22(5), 1410-1416.

Bub, D. N., Masson, M. E., \& Lalonde, C. E. (2006). Cognitive Control in Children: Stroop Interference and Suppression of Word Reading. Psychological Science, 17(4), 351357. doi:10.1111/j.1467-9280.2006.01710.x

Burle, B., Spieser, L., Servant, M., \& Hasbroucq, T. (2014). Distributional reaction time properties in the eriksen task: Marked differences or hidden similarities with the simon task? Psychonomic Bulletin \& Review, 21(4), 1003-1010.

Davranche, K., Casini, L., Arnal, P. J., Rupp, T., Perrey, S., \& Verges, S. (2016). Cognitive functions and cerebral oxygenation changes during acute and prolonged hypoxic exposure. Physiology \& Behavior, 164, 189-197.

Davranche, K., Hall, B., \& McMorris, T. (2009). Effect of acute exercise on cognitive control required during an Eriksen flanker task. Journal of Sport and Exercise Psychology, 31(5), 628-639.

De Jong, R., Liang, C.-C., \& Lauber, E. (1994). Conditional and unconditional automaticity: A dual-process model of effects of spatial stimulus-response correspondence. Journal of Experimental Psychology: Human Perception and Performance, 20(4), 731.

de Bruin, A., \& Della Sala, S. (2017). Effects of age on inhibitory control are affected by task-specific features. The Quarterly Journal of Experimental Psychology, (71), 1219-1233.

Debey, E., Ridderinkhof, R. K., De Houwer, J., De Schryver, M., \& Verschuere, B. (2015). Suppressing the truth as a mechanism of deception: Delta plots reveal the role of 
response inhibition in lying. Consciousness and Cognition, 37, 148-159. doi:10.1016/ j.concog.2015.09.005

Donders, F. (1868). On the speed of mental processes; transl. Koster, W. In Attention and performance II: Proceedings of the Donders centenary symposiumon reaction time, held in Eindhoven july 29-august 2, 1968, North-Holland Publishing.

Eimer, M. (1999). Facilitatory and inhibitory effects of masked prime stimuli on motor activation and behavioural performance. Acta Psychologica, 101 (2-3), 293-313.

Finkbeiner, M., \& Caramazza, A. (2008). Modulating the masked congruence priming effect with the hands and the mouth. Journal of Experimental Psychology: Human Perception and Performance, 34 (4), 894.

Forstmann, B. U., van den Wildenberg, W. P., \& Ridderinkhof, K. R. (2008). Neural mechanisms, temporal dynamics, and individual differences in interference control. Journal of Cognitive Neuroscience, 20(10), 1854-1865.

Gajdos, T. (2002). Measuring inequalities without linearity in envy: Choquet integrals for symmetric capacities. Journal of Economic Theory, 106, 190-200.

Gini, C. (1912). Variabilità e mutabilitaà. Bologna.

Hommel, B. (1993). The relationship between stimulus processing and response selection in the simon task: Evidence for a temporal overlap. Psychological research, 55(4), $280-290$.

Hommel, B. (1994). Spontaneous decay of response-code activation. Psychological research, $56(4), 261-268$.

Hübner, R., \& Töbel, L. (2019). Conflict resolution in the eriksen flanker task: Similarities and differences to the simon task. PloS one, $14(3)$, e0214203.

Hyndman, R. J., \& Fan, Y. (1996). Sample quantiles in statistical packages. The American Statistician, 50(4), 361-365.

Joyce, J., Smyth, P. J., Donnelly, A. E., \& Davranche, K. (2014). The Simon Task and Aging: Does Acute Moderate Exercise Influence Cognitive Control? Medicine 63 Science in Sports \& Exercise, 46(3), 630-639. doi:10.1249/MSS.0b013e3182a77980

Kruschke, J. K. (2013). Bayesian estimation supersedes the t test. Journal of Experimental Psychology: General, 142(2), 573.

Kruschke, J. K., \& Meredith, M. (2018). Best: Bayesian estimation supersedes the t-test. R package version 0.5.1. Retrieved from https://CRAN.R-project.org/package $=$ BEST

Lorenz, M. O. (1905). Methods of Measuring the Concentration of Wealth. Publications of the American Statistical Association, 9(70), 209. doi:10.2307/2276207

Luce, R. D. (1986). Response times: Their role in inferring elementary mental organization. Oxford University Press on Demand.

Mattler, U. (2003). Delayed flanker effects on lateralized readiness potentials. Experimental Brain Research, 151(2), 272-288.

McBride, J., Sumner, P., Jackson, S. R., Bajaj, N., \& Husain, M. (2013). Exaggerated object affordance and absent automatic inhibition in alien hand syndrome. Cortex, 49(8), 2040-2054.

Meier, M. E., \& Kane, M. J. (2015). Carving executive control at its joints: Working memory capacity predicts stimulus-stimulus, but not stimulus-response, conflict. 
Journal of Experimental Psychology: Learning, Memory, and Cognition, 41(6), 18491872.

Pratte, M. S., Rouder, J. N., Morey, R. D., \& Feng, C. (2010). Exploring the differences in distributional properties between Stroop and Simon effects using delta plots. Attention, Perception \& Psychophysics, 72(7), 2013-2025. doi:10.3758/APP.72.7.2013

Proctor, R. W., Miles, J. D., \& Baroni, G. (2011). Reaction time distribution analysis of spatial correspondence effects. Psychonomic Bulletin \& Review, 18(2), 242-266. doi:10.3758/s13423-011-0053-5

Ratcliff, R. (1978). A theory of memory retrieval. Psychological review, 85(2), 59.

Ridderinkhof, R. K. (2002a). Activation and suppression in conflict tasks: Empirical clarification through distributional analyses. Attention and performance XIX: Common mechanisms in perception and action, 494-519.

Ridderinkhof, R. K. (2002b). Micro-and macro-adjustments of task set: Activation and suppression in conflict tasks. Psychological research, 66 (4), 312-323.

Ridderinkhof, R. K., Scheres, A., Oosterlaan, J., \& Sergeant, J. A. (2005). Delta Plots in the Study of Individual Differences: New Tools Reveal Response Inhibition Deficits in AD/HD That Are Eliminated by Methylphenidate Treatment. Journal of Abnormal Psychology, 114 (2), 197-215. doi:10.1037/0021-843X.114.2.197

Schwarz, W., \& Miller, J. (2012). Response time models of delta plots with negative-going slopes. Psychonomic Bulletin \& Review, 19(4), 555-574. doi:10.3758/s13423-0120254-6

Servant, M., Gajdos, T., \& Davranche, K. (2018). ELF: A new measure of response capture. Psychonomic Bulletin \& Review, 25, 539-547.

Servant, M., White, C., Montagnini, A., \& Burle, B. (2016). Linking theoretical decisionmaking mechanisms in the simon task with electrophysiological data: A model-based neuroscience study in humans. Journal of Cognitive Neuroscience, 28(10), 15011521.

Sharma, D., Booth, R., Brown, R., \& Huguet, P. (2010). Exploring the temporal dynamics of social facilitation in the stroop task. Psychonomic Bulletin $\mathscr{E}$ Review, 17(1), 5258.

Simon, J. R. (1969). Reactions toward the source of stimulation. Journal of Experimental Psychology, 81 (1), 174-176. doi:10.1037/h0027448

Suarez, I., Burle, B., Tobon, C., Pineda, D., Lopera, F., Hasbroucq, T., \& Casini, L. (2015). Deciphering interference control in adults with ADHD by using distribution analyses and electromyographic activity. Acta Psychologica, 159, 85-92.

Ulrich, R., Schröter, H., Leuthold, H., \& Birngruber, T. (2015). Automatic and controlled stimulus processing in conflict tasks: Superimposed diffusion processes and delta functions. Cognitive psychology, 78, 148-174.

van den Wildenberg, W. P. M., Wylie, S. A., Forstmann, B. U., Burle, B., Hasbroucq, T., Ridderinkhof, R. K., et al. (2010). To head or to heed? Beyond the surface of selective action inhibition: A review. Frontiers in Human Neuroscience, 4(222).

van Wouwe, N. C., Kanoff, K. E., Claassen, D. O., Spears, C. A., Neimat, J., van den Wildenberg, W. P. M., \& Wylie, S. A. (2016). Dissociable effects of dopamine on the 
initial capture and the reactive inhibition of impulsive actions in parkinson's disease. Journal of Cognitive Neuroscience, 28(5), 710-723. doi:10.1162/jocn_a_00930

Wagenmakers, E.-J., \& Brown, S. (2007). On the linear relation between the mean and the standard deviation of a response time distribution. Psychological Review, 114(3), 830-841. doi:10.1037/0033-295X.114.3.830

White, C. N., Servant, M., \& Logan, G. (2017). Practical considerations for using conflictbased diffusion models to interpret choice RT data. Psychonomic Bulletin $\mathcal{E}$ Review. in press.

Wylie, S. A., Ridderinkhof, K. R., Elias, W. J., Frysinger, R. C., Bashore, T. R., Downs, K. E., ... van den Wildenberg, W. P. (2010). Subthalamic nucleus stimulation influences expression and suppression of impulsive behaviour in parkinson's disease. Brain, 133(12), 3611-3624.

Wylie, S. A., Claassen, D. O., Huizenga, H. M., Schewel, K. D., Ridderinkhof, R. K., Bashore, T. R., \& van den Wildenberg, W. P. M. (2012). Dopamine agonists and the suppression of impulsive motor actions in Parkinson disease. Journal of Cognitive Neuroscience, 24 (8), 1709-1724.

Wylie, S. A., Claassen, D. O., Kanoff, K. E., Ridderinkhof, K. R., \& van den Wildenberg, W. P. (2013). Impaired inhibition of prepotent motor actions in patients with tourette syndrome. Journal of psychiatry 83 neuroscience: JPN, 38(5), 349.

Wylie, S. A., Ridderinkhof, K. R., Eckerle, M. K., \& Manning, C. A. (2007). Inefficient response inhibition in individuals with mild cognitive impairment. Neuropsychologia, 45(7), 1408-1419.

Wylie, S. A., Van Den Wildenberg, W., Ridderinkhof, K., Bashore, T., Powell, V., Manning, C., \& Wooten, G. (2009). The effect of speed-accuracy strategy on response interference control in parkinson's disease. Neuropsychologia, 47(8-9), 1844-1853.

Zhang, J., \& Kornblum, S. (1997). Distributional analysis and De Jong, Liang, and Lauber's (1994) dual-process model of the Simon effect. Journal of Experimental Psychology: Human Perception and Performance, 23(5), 1543-1551. doi:10.1037/00961523.23.5.1543 